Biogeosciences, 7, 43-55, 2010

www.biogeosciences.net/7/43/2010/

(c) Author(s) 2010. This work is distributed under

the Creative Commons Attribution 3.0 License.

\title{
Regional and seasonal patterns of litterfall in tropical South America
}

\author{
J. Chave ${ }^{1}$, D. Navarrete ${ }^{2,3}$, S. Almeida ${ }^{4}$, E. Álvarez ${ }^{3}$, L. E. O. C. Aragão ${ }^{5}$, D. Bonal ${ }^{6}$, P. Châtelet ${ }^{7}$, J. E. Silva-Espejo ${ }^{8}$, \\ J.-Y. Goret ${ }^{6}$, P. von Hildebrand ${ }^{2}$, E. Jiménez ${ }^{3}$, S. Patiño ${ }^{3}$, M. C. Peñuela ${ }^{3}$, O. L. Phillips ${ }^{9}$, P. Stevenson ${ }^{10}$, and Y. Malhi ${ }^{5}$ \\ ${ }^{1}$ Laboratoire Evolution et Diversité Biologique, UMR 5174 CNRS/UPS, Toulouse, France \\ ${ }^{2}$ Fundación Puerto Rastrojo, Bogotá, Colombia \\ ${ }^{3}$ Grupo de Estudio de Ecosistemas Terrestres Tropicales, Universidad Nacional de Colombia, Leticia, Colombia \\ ${ }^{4}$ Museu Paraense Emilio Goeldi, 66077-530 Belem, Brazil \\ ${ }^{5}$ Environmental Change Institute, School of Geography and the Environment, University of Oxford, South Parks Road, \\ Oxford OX1 3QY, UK \\ ${ }^{6}$ INRA, UMR Ecologie des Forêts de Guyane, BP 709, 97387 Kourou Cedex, French Guiana \\ ${ }^{7}$ CNRS-Guyane, Station d'Etude des Nouragues, UPS 2561, French Guiana \\ ${ }^{8}$ Universidad San Antonio Abad, Cusco, Perú \\ ${ }^{9}$ Earth and Biosphere Institute, School of Geography, University of Leeds, Leeds LS2 9JT, UK \\ ${ }^{10}$ Universidad de los Andes, Bogotá, Colombia
}

Received: 16 December 2008 - Published in Biogeosciences Discuss.: 27 July 2009

Revised: 4 December 2009 - Accepted: 4 December 2009 - Published: 5 January 2010

\begin{abstract}
The production of aboveground soft tissue represents an important share of total net primary production in tropical rain forests. Here we draw from a large number of published and unpublished datasets $(n=81$ sites) to assess the determinants of litterfall variation across South American tropical forests. We show that across old-growth tropical rainforests, litterfall averages $8.61 \pm 1.91 \mathrm{Mg} \mathrm{ha}^{-1} \mathrm{yr}^{-1}$ (mean \pm standard deviation, in dry mass units). Secondary forests have a lower annual litterfall than old-growth tropical forests with a mean of $8.01 \pm 3.41 \mathrm{Mg} \mathrm{ha}^{-1} \mathrm{yr}^{-1}$. Annual litterfall shows no significant variation with total annual rainfall, either globally or within forest types. It does not vary consistently with soil type, except in the poorest soils (white sand soils), where litterfall is significantly lower than in other soil types $\left(5.42 \pm 1.91 \mathrm{Mg} \mathrm{ha}^{-1} \mathrm{yr}^{-1}\right)$. We also study the determinants of litterfall seasonality, and find that it does not depend on annual rainfall or on soil type. However, litterfall seasonality is significantly positively correlated with rainfall seasonality. Finally, we assess how much carbon is stored in reproductive organs relative to photosynthetic organs. Mean leaf fall is $5.74 \pm 1.83 \mathrm{Mg} \mathrm{ha}^{-1} \mathrm{yr}^{-1}$ (71\% of
\end{abstract}

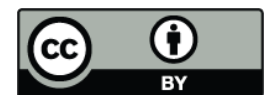

Correspondence to: J. Chave (chave@ cict.fr) total litterfall). Mean allocation into reproductive organs is $0.69 \pm 0.40 \mathrm{Mg} \mathrm{ha}^{-1} \mathrm{yr}^{-1}$ (9\% of total litterfall). The investment into reproductive organs divided by leaf litterfall increases with soil fertility, suggesting that on poor soils, the allocation to photosynthetic organs is prioritized over that to reproduction. Finally, we discuss the ecological and biogeochemical implications of these results.

\section{Introduction}

Since the early 1950s, an enormous amount of research has been devoted to the measurement of net primary production (NPP) in ecosystems, the amount of carbon that is fixed from the atmosphere into new organic matter. Of the 720 references reported in the Osnabrïck dataset (Esser et al., 1997), only 21 were collected in tropical forest environments, an astonishingly small figure given that tropical rainforests account for a third of global terrestrial NPP, and savannas another quarter (Grace, 2004). Since that time, much progress has been made to quantify the carbon cycle in tropical forest ecosystems (Malhi et al., 2002, 2009; Keller et al., 2004), and there is still much activity around the development of global databases of the carbon cycle in terrestrial environments (Luyssaert et al., 2007).

Published by Copernicus Publications on behalf of the European Geosciences Union. 
In one of the most thorough recent reappraisals of tropical forest NPP quantification, Clark et al. (2001) compiled data from 39 tropical forest sites and they estimated total tropical forest NPP. Their estimates ranged between 3.1 and 21.7 $\mathrm{Mg} \mathrm{ha}^{-1} \mathrm{yr}^{-1}$, of which, 1.8 to $12.0 \mathrm{Mg} \mathrm{ha}^{-1} \mathrm{yr}^{-1}$ were allocated into soft tissues (leaves, reproductive organs and twigs). Tropical forest NPP was found to be poorly correlated with mean annual temperature and with annual rainfall (see also Schuur, 2003; Del Grosso et al., 2008). In a previous contribution, Malhi et al. (2004) explored the regional variation of the fraction of carbon fixed aboveground into woody parts in tropical South America (trunks and branches, wNPP). They focused on 104 permanent sampling plots where trunk diameter had been measured several times, and estimated the annual amount of carbon fixed into wood. Their major finding was that wNPP varied dramatically at the regional scale, and that a large part of this regional variation was due to soil type. Using the data available at 10 tropical forest sites in Amazonia, Aragão et al. (2009) showed that total NPP ranged between 18.6 and $34.0 \mathrm{Mg} \mathrm{ha}^{-1} \mathrm{yr}^{-1}$, with a mean of $25.6 \mathrm{Mg} \mathrm{ha}^{-1} \mathrm{yr}^{-1}$, much greater than recent regional tropical forest estimates (e.g. Luyssaert et al., 2007; Del Grosso et al., 2008).

Clark et al. (2001) also suggested that NPP was not strongly correlated with total litterfall, as had been previously suggested by Bray and Gorham's (1964) global model. They however acknowledged that their estimates were based on an indirect estimation of several key components of NPP. For Amazonian forests, Aragão et al. (2009) provide a most useful perspective on this question. Their analysis strongly supports Bray and Gorham's (1964) model: total NPP is consistently close to 3.1 times total litterfall. If their finding is general, this is a strong motivation for summarizing our current knowledge on the regional and temporal variation of total litterfall in the Amazon.

In the present contribution, we focus on the amount of carbon fixed into organs with short residence time, such as leaves, reproductive organs (flowers, fruits), and small branches. Like in most previous analyses, we assume that the ecosystem is at equilibrium, that is, the flux of carbon into this pool of carbon equals the flux of carbon outside of this flux. Then, the amount of NPP allocated annually to leaves, reproductive organs, and small branches should be equal to the annual litterfall. Leaf production and other components of litterfall should depend upon a large suite of environmental and geographical factors. In tropical South America, the determinants of this spatial variation remain poorly studied, and it is impossible to get even a superficial sense of the changes in litterfall production across environments and across regions. The goal of the present manuscript is to review the recent literature and explore whether available data are sufficient to draw general rules for the spatial variation of litterfall across South America.
We here bring together a large number of published and unpublished litterfall datasets, including a wide range of environmental conditions, such as terra firme rainforests, flooded rainforests, dry forests, and montane forests. We also partition litterfall into its main three components (leaves, fruits and flowers, and twigs, see Proctor, 1983). We use this dataset to assess what determines the spatial and temporal variability in litterfall. Specifically, we address the following questions: (1) Is annual litterfall determined by edaphic or climatic factors? (2) Is the seasonality of litterfall determined by edaphic or climatic factors?, and (3) Does plant investment into photosynthetic organs and reproductive organs depend on environmental factors? Finally, we discuss the implications of our findings.

\section{Methods}

\subsection{Dataset}

We combed the literature for publications reporting figures on litterfall in tropical South America. In our analysis, we included the studies in central Panama, but not those of the rest of Central America. We also included a number of unpublished data. For each study, we reported the different parts of litterfall, including leaves, branches (usually less than $2 \mathrm{~cm}$ in diameter), flowers, fruits, and others, if available (Proctor, 1983). Litterfall was collected in litter-traps set up ca. 1-2 $\mathrm{m}$ above the ground to avoid disturbance by large mammals. We recorded the duration of the experiment, number of traps, and size of the traps. All litterfall figures (annual and monthly) were converted into $\mathrm{Mg} \mathrm{ha}^{-1} \mathrm{yr}^{-1}$ of dry biomass. We did not correct these figures for a possible loss to herbivory between censuses (Leigh, 1999; Clark et al., 2001), because this would have entailed making additional uncontrolled assumptions. Our litterfall estimates did not incorporate coarse woody debris, which may account for a sizeable fraction of carbon loss from the live vegetation (Chambers et al., 2001; Nepstad et al., 2002). In most cases, these estimates did not incorporate palm leaves which tend to be too large to be trapped by litter-traps, and the fruits and leaves produced by understory plants. This may result in a significant under-estimation of litterfall. For instance, in a wet rain forest of Costa Rica, over $10 \%$ of the total leaf area was below $2 \mathrm{~m}$ above ground (Clark et al., 2008).

In total, we report on 29 published studies (64 sites) and 7 unpublished ones (17 sites). The 81 sites included in the present analysis are detailed in Table 1. All of these studies comply with the minimal conditions for litterfall sampling proposed by Proctor (1983). The sampling duration varied from 1 year to 7 years (mean across sites: $1.97 \mathrm{yr}$ ), and the total area sampled (number of litterfall traps multiplied by the size of these traps, in $\mathrm{m}^{2}$ ) varied from 1.92 to $60 \mathrm{~m}^{2}$ (mean across sites: $10.1 \mathrm{~m}^{2}$ ), with each trap at least $0.25 \mathrm{~m}^{2}$ in area. 


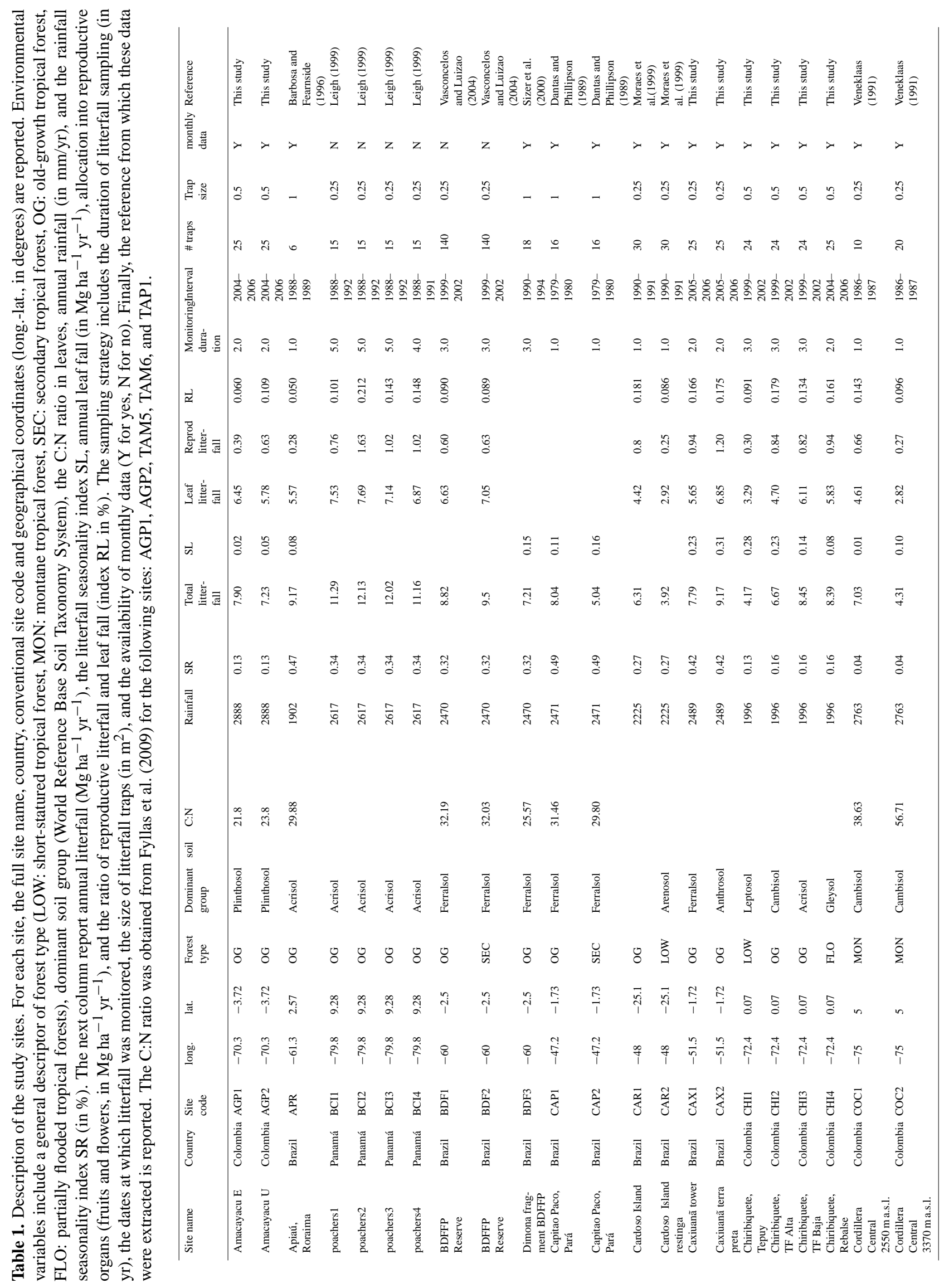




\begin{tabular}{|c|c|c|c|c|c|c|c|c|c|c|c|c|c|c|c|c|c|c|c|c|c|c|c|c|c|}
\hline 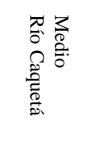 & 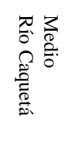 & 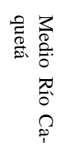 & 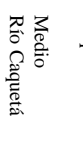 & 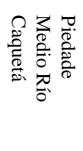 & & & & & & & 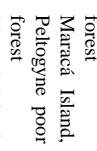 & 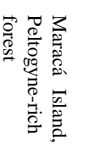 & & & & & & & & & & & & 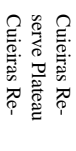 & \\
\hline $\begin{array}{l}\frac{\delta}{0} \\
\frac{0}{\overline{2}} \\
\frac{2}{2}\end{array}$ & $\begin{array}{l}\frac{\delta}{\frac{\delta}{2}} \\
\frac{\bar{z}}{\tilde{z}}\end{array}$ & 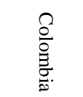 & 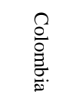 & 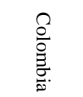 & 莺 & 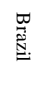 & & 芯 & 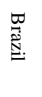 & 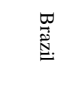 & : & 薦 & 莽 & 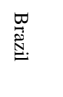 & 萝 & 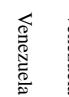 & & 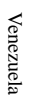 & 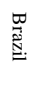 & 蔍 & 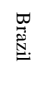 & 㠇 & 萢 & 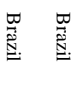 & 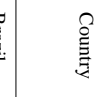 \\
\hline 呇 & 疍 & 诵 & 疍 & 疍 & 䍘 & $\underset{\Xi}{3}$ & 壳 & 旁 & 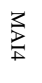 & 㱏 & 总 & 声 & $\underset{\nearrow}{\Xi}$ & 总 & 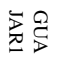 & 恿 & 恿 & 号 & $\stackrel{\vec{g}}{\check{c}}$ & $\xi$ & ఫ్ర్心 & 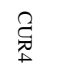 & ఫ్ర్రి & 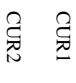 & $\frac{\delta}{\partial}$ \\
\hline$\underset{\substack{1 \\
N}}{\substack{n \\
i n}}$ & 悹 & $\underset{\substack{N \\
ن}}{1}$ & $\underset{\substack{n \\
心}}{i}$ & 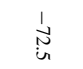 & $\underset{\mathrm{i}}{\stackrel{d}{u}}$ & $\underset{\mathrm{N}}{\stackrel{d}{\sim}}$ & 占 & 恧 & $\frac{1}{9}$ & $\stackrel{\frac{1}{g}}{\dot{q}}$ & $\stackrel{\frac{1}{a}}{\frac{1}{b}}$ & $\frac{b}{d}$ & $\underset{\substack{\dot{\infty} \\
\infty \\
\infty}}{1}$ & 岕 & 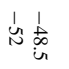 & $\frac{1}{\grave{\omega}}$ & $\stackrel{\frac{1}{\omega}}{\grave{\omega}}$ & $\frac{1}{2}$ & $\begin{array}{l}\dot{b}_{b} \\
\underset{\infty}{\infty}\end{array}$ & $\stackrel{\dot{b}}{\stackrel{4}{+}}$ & d. & $\stackrel{d}{d}$ & do & $\begin{array}{ll}1 & 1 \\
0 & 0 \\
: & 0\end{array}$ & 8 \\
\hline $\begin{array}{l}1 \\
\text { d } \\
\text { 辛 }\end{array}$ & $\begin{array}{l}b \\
\dot{d} \\
\text { d }\end{array}$ & $\begin{array}{l}b \\
\dot{d} \\
\text { i }\end{array}$ & $\begin{array}{l}1 \\
\dot{d} \\
\dot{t}\end{array}$ & $\begin{array}{l}1 \\
\dot{b} \\
\text { i }\end{array}$ & $\underset{\infty}{\grave{\infty}}$ & $\underset{\omega}{\stackrel{1}{\infty}}$ & $\stackrel{\underline{\omega}}{\omega}$ & 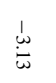 & 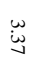 & 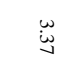 & $\stackrel{\omega}{\omega_{u}}$ & $\stackrel{w}{\dot{\sim}}$ & $\begin{array}{l}1 \\
\\
\dot{+}\end{array}$ & $\perp$ & $1 \frac{1}{\stackrel{\omega}{\lrcorner}}$ & u & u & is & î̀ & $\stackrel{i}{n}$ & 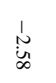 & 岂 & 点 & 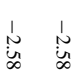 & . \\
\hline 8 & 8 & 8 & 8 & 꽁 & 哲 & 8 & 腤 & 8 & 꽁 & 꽁 & 꽁 & 꽁 & $\&$ & 吕 & ৪৪ & $\underset{\&}{5}$ & \& & 8 & 8 & 8 & 꽁 & 8 & $\&$ & 꽁 \& & 志哥 \\
\hline 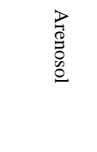 & 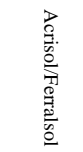 & 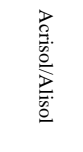 & 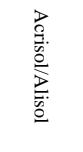 & 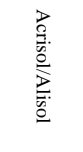 & 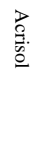 & $\begin{array}{l}\text { 竞 } \\
\text { 产. } \\
\underline{\underline{n}}\end{array}$ & 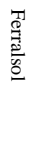 & 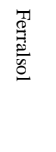 & 总 & 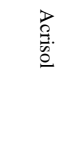 & 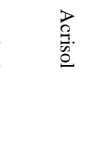 & 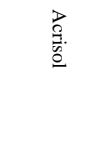 & 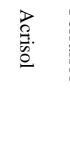 & 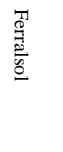 & 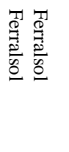 & 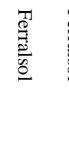 & 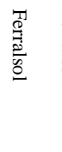 & 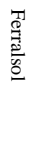 & 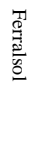 & 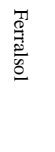 & 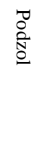 & $\begin{array}{l}\text { 童 } \\
\text { 峷 }\end{array}$ & 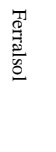 & 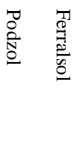 & 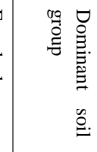 \\
\hline$\underset{\substack{ \pm \infty}}{ \pm}$ & 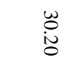 & $\stackrel{\breve{\omega}}{\dot{\omega}}$ & :ّ & 葛 & & & 苞 & $\frac{\omega}{\dot{\sigma}}$ & $\begin{array}{l}\text { u } \\
\text { ț }\end{array}$ & 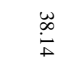 & 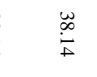 & 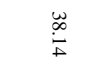 & & & 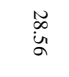 & $\frac{u}{6}$ & 剀 & 㟔 & $\stackrel{\omega}{\Xi}$ & 岕 & 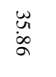 & 芯 & 䓵 & 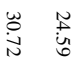 & \\
\hline$\underset{\mathscr{N}}{\mathbb{N}}$ & $\underset{\mathscr{N}}{\mathbb{N}}$ & $\underset{\mathscr{N}}{\mathbb{N}}$ & 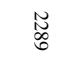 & $\underset{\substack{\mathbb{O} \\
\mathbb{N}}}{\mathbb{N}}$ & 商 & 芯 & $\frac{N}{0}$ & $\frac{N}{\sigma}$ & 讨 & $\overrightarrow{\mathrm{N}}$ & $\overrightarrow{\mathbb{N}}$ & $\overline{\underline{N}}$ & $\overrightarrow{\mathrm{g}}$ & 谂 & 颉 & $\overrightarrow{\breve{s}}$ & $\overline{\underline{y}}$ & $\overrightarrow{\mathrm{s}}$ & 兽 & $\vec{\Xi}$ & 等 & 莣 & 等 & 羔莣 & 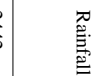 \\
\hline : & : & : & : & : & i & 迹 & 总 & 兽 & 总 & 总 & fo & 总 & 잉 & : & 㫣是 & ¿ & : & : & i⿱宀 & 总 & $\bigodot_{+}$ & $\sum_{+}$ & 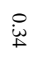 & $\underset{i}{\dot{i}}$ & 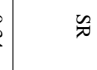 \\
\hline iे & $\stackrel{\infty}{\infty}$ & $\stackrel{\infty}{\infty}$ & 8 & $\vec{i}$ & $\underset{\stackrel{F}{f}}{F}$ & $\underset{\sim}{\stackrel{N}{\sim}}$ & $\underset{\infty}{\vec{\infty}}$ & $\stackrel{\infty}{\unlhd}$ & i & $\begin{array}{l}\infty \\
\infty \\
\infty \\
\infty\end{array}$ & $\dot{\grave{j}}$ & $\vec{\omega}$ & $\underset{\infty}{\vec{\infty}}$ & $\begin{array}{l}\infty \\
\dot{4} \\
\dot{4}\end{array}$ & $\underset{\vec{t}}{\overrightarrow{\vec{t}}} \stackrel{0}{0}$ & $\underset{\omega}{\omega}$ & $\tilde{a}$ & $\frac{0}{6}$ & $\vec{\omega}$ & $\stackrel{\bullet}{\sim}$ & oे & $\vec{\sigma}$ & 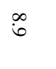 & $\vec{\perp}$ & 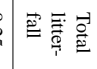 \\
\hline & & & & & i & i & $\stackrel{i}{i}$ & $\stackrel{\circ}{u}$ & $:$ & : & 总 & ì & : & $\frac{8}{6}$ & ĩ & $\stackrel{\circ}{\circ}$ & $\stackrel{\circ}{\infty}$ & $\stackrel{\circ}{\infty}$ & & $\stackrel{0}{i n}$ & & & & $\stackrel{8}{\circ}$ & 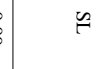 \\
\hline 幽 & $\ddot{u}$ & $\dot{\vec{y}}$ & $\dot{\hat{z}}$ & $\ddot{\check{\sigma}}$ & $\overrightarrow{0}$ & 总 & : & :े & is & 范 & iे & $\breve{\ddagger}$ & $\ddot{8}$ & ì & $\begin{array}{l}1 \\
\infty \\
\infty\end{array}$ & & & & $\ddot{g}$ & के & $\underset{\substack{\infty \\
\infty}}{+}$ & $\dot{\alpha}$ & 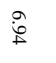 & 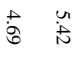 & 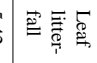 \\
\hline 8 & 讪 & \& & 总 & $\dot{i n}$ & نे & 总 & $\stackrel{巳}{\dot{\rho}}$ & \& & $\overline{\mathrm{v}}$ & : & 总 & $\stackrel{\ominus}{\exists}$ & & 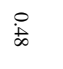 & $\bar{\sigma}$ & & & & 总 & $\overline{\grave{s}}$ & & & & & $\vec{P}$ \\
\hline 总 & $\stackrel{\circ}{\circ}$ & 离 & : & 总 & $\dot{\circ}$ & 兽 & 总 & : & 总 & $\stackrel{8}{\mathrm{y}}$ & $\stackrel{P}{\stackrel{\sim}{\omega}}$ & $\stackrel{8}{\underline{\Xi}}$ & & 高 & $\stackrel{8}{\stackrel{D}{\infty}}$ & & & & 离 & $\stackrel{\dot{0}}{\infty}$ & & & & 总 & $\pi$ \\
\hline 5 & $\overline{0}$ & 5 & $\bar{\circ}$ & $\vec{\circ}$ & 5 & $\vec{\circ}$ & $\stackrel{n}{0}$ & $\stackrel{n}{\circ}$ & $\ddot{\circ}$ & है & 5 & $\vec{\circ}$ & $\vec{\circ}$ & 5 & 5 & $\vec{\circ}$ & $\overrightarrow{0}$ & $\dot{0}$ & $\vec{\circ}$ & $\bar{\circ}$ & & & & $\stackrel{\omega}{\circ}$ & 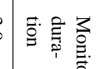 \\
\hline 兽宫 & 宽 & 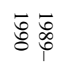 & 宛 & 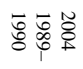 & 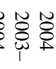 & 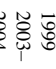 & : & 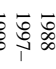 & 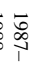 & 惫兽 & 悹兽 & 总总尊 & 落总总 & 绰总 & & & 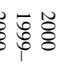 & & 要 & 定 & & & & 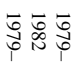 & בె \\
\hline $\bar{u}$ & $\vec{u}$ & $\bar{u}$ & $\bar{u}$ & $\bar{u}$ & $\bar{\sigma}$ & $\overline{0}$ & $\tilde{8}$ & $\tilde{8}$ & N & $\stackrel{\omega}{\omega}$ & $\stackrel{\omega}{\omega}$ & $\stackrel{\omega}{\omega}$ & $\bar{u}$ & $\overline{8}$ & $\overline{8}$ & $\infty$ & $\infty$ & $\infty$ & $\overline{0}$ & 岕 & $\overline{0}$ & $\bar{\sigma}$ & & $\vec{u} \quad \bar{u}$ & \\
\hline 怘 & 总 & 总 & 总 & 总 & 总 & 总 & 总 & 总 & - & 怘 & 总 & 总 & & 总 & ن & in & in & in & 总 & - & 总 & 总 & 总 & in in & 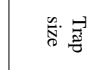 \\
\hline$z$ & z & z & z & $z$ & $\prec$ & $\prec$ & . & $\prec$ & $\prec$ & $\prec$ & $\prec$ & $\prec$ & $\prec$ & $\prec$ & & $\prec$ & $\prec$ & 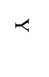 & z & & z & $z$ & z & $\prec \prec$ & 䜌 \\
\hline 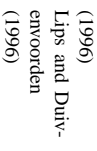 & 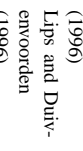 & 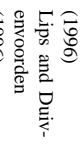 & 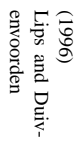 & 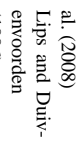 & & & & 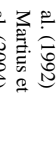 & & 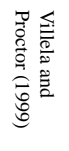 & 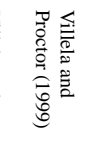 & 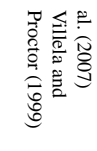 & & & & & 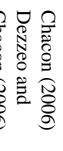 & & & & & 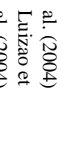 & & 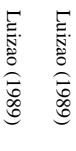 & \\
\hline
\end{tabular}




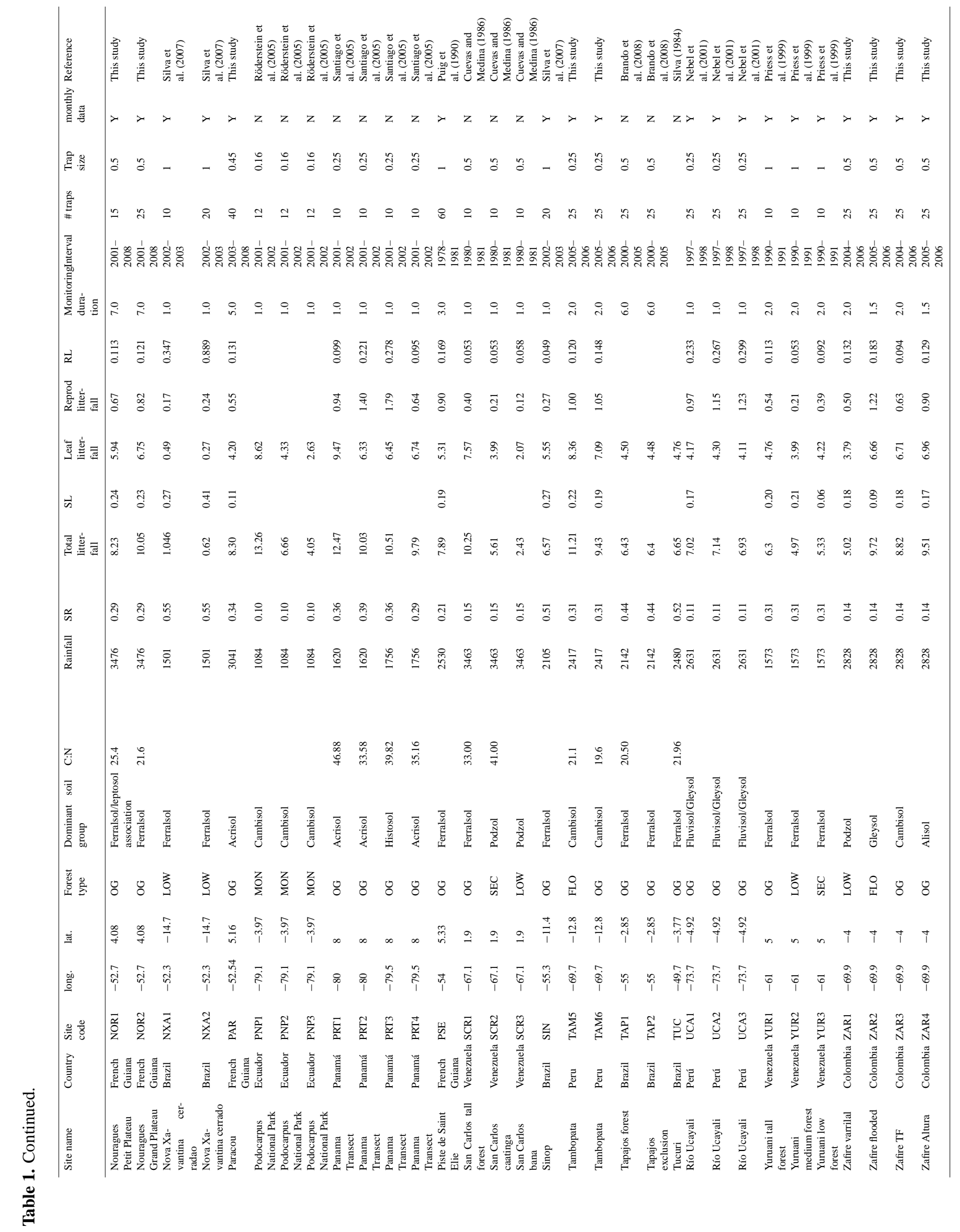


To evaluate the seasonality of litterfall, we created a database including the monthly litterfall data as reported in the published reports or in unpublished datasets. In a number of cases, these figures were reported in the form of figures. We scanned the figures, and retrieved the original data by digitizing the figure manually using the software DigitizeIt, version 1.5.8 (http://www.digitizeit.de/).

\subsection{Environmental variables}

Environmental variables included in the present analysis are soil type (see also Malhi et al., 2004), and rainfall data. Soil type, when available, was deduced from the publications, and mostly based on the World Reference Base Soil Taxonomy (WRB, 2006). More details on the distribution, area, and chemical properties of these soils type in Amazonia are available in Quesada (2009, see also Quesada et al., 2009). We classified the sites into four main soil categories, roughly increasing in soil fertility (concentration of phosphorus and of exchangeable cations in the soil, Quesada et al., 2009): A) highly permeable infertile soils (arenosols and podzols); B) relatively infertile ancient soils (ferrasols); C) relatively fertile acidic soils (acrisols, plinthosols and alisols) and D) fertile young or wet soils (cambisols, leptosols, histosols, gleysols or fluvisols). The one site with humanderived soil (archeo-anthrosol, CAX2 site: terra preta) was excluded from this classification.

When possible, we also reported the concentration of nitrogen in litterfall $(\mathrm{N}, \mathrm{P})$. The carbon to nitrogen ratio $(\mathrm{C}: \mathrm{N}$ ratio) measure the depletion of nitrogen in plants. This value is correlated with the resource availability of the soil on which the plants grow (McGroddy et al., 2004; Ågren, 2008; Quesada, 2009). If only data on N concentrations were available in live leaves (see e.g. Fyllas et al., 2009), we made use of these figures instead to compute the $\mathrm{C}: \mathrm{N}$ ratio. We did not have enough values of phosphorus concentration in the litter to measure the $\mathrm{N}: \mathrm{P}$ ratio, and estimating the litter $\mathrm{P}$ concentration from green leaf $\mathrm{P}$ concentration is difficult, because $\mathrm{P}$ is massively retranslocated before leaf abscission (Chuyong et al., 2000). Hättenschwiler et al. (2008) show that there is no correlation between green leaf N:P and litter N:P.

Rainfall was derived from a climatic dataset that covers the period 1960-1998, obtained by interpolating among local meteorological stations, and correcting apparently erroneous data (New et al., 1999). This dataset reproduces well the observed gradients in rainfall over the Amazon. For a few sites with steep climatic gradients near the Andes or close to the oceans, local meteorological data were preferred.

We also classified the data by forest type. The majority ( $n=51$ ) was old-growth tropical rain forest (OG), but we also included a number of secondary (i.e. recently disturbed) rain forests (SEC, $n=7$ ), periodically or permanently flooded rainforest (FLO, $n=10$ ), montane rainforests (MON, $n=5$ ), and low vegetation (LOW, $n=7$ ). This last category is a composite of different vegetation types, includ- ing low vegetation growing on Colombian tepuis (Chiribiquete National Park), woodland savannas in Brazil and Colombia (cerrado), coastal oceanic vegetation in Brazil (restinga), and stunted forest in Venezuela (caatinga).

\subsection{Statistical analyses}

We computed an index of seasonality as follows. We converted the month into a number from 0 (1 January) to 330 (1 December). This represents the number of days elapsed since the beginning of the year but also an angle in degrees. We used this convention to represent the data using a polar plot (Fig. 1), where the litterfall of month $i$ are plotted using a vector starting from $(0,0)$, with a length equal to the litterfall at month $i$ (in $\mathrm{Mg} \mathrm{ha}^{-1} \mathrm{yr}^{-1}$ ) and the angle equal to 30*i (in degrees). The mean vector is obtained from the average of the projections along the $\mathrm{x}$ and the $\mathrm{y}$ axes. A similar analysis was performed to study the patterns of phenology across two seasonal rainforests (Zimmerman et al., 2007). The mathematical definition of the mean vector, $\boldsymbol{m}=\left(m_{x}, m_{y}\right)$, from the 12 monthly litterfall vectors $\boldsymbol{L}^{i}$ is:

$m_{x}=\frac{1}{12} \sum_{i=0}^{11} L^{i} \cos (30 \times i), \quad m_{y}=\frac{1}{12} \sum_{i=0}^{11} L^{i} \sin (30 \times i)$

Here, $L^{i}=\left\|\boldsymbol{L}^{i}\right\|$ is the absolute value of litterfall (in $\mathrm{Mg} \mathrm{ha}^{-1} \mathrm{yr}^{-1}$ ) for month $i$. Using these definition, annual litterfall is $L=\sum_{i=0}^{11} L^{i} / 12$. We finally define the seasonality index as follows

$\mathrm{SL}=\frac{\|\boldsymbol{m}\|}{L}$

This index measures whether litterfall is evenly distributed throughout the year, in which case $\mathrm{SL} \approx 0$. Alternatively, if litter falls only during one month, then $\mathrm{SL} \approx 1$. Figure 1 represents polar plots with monthly litterfall data and the location of the mean vector, $\boldsymbol{m}=\left(m_{x}, m_{y}\right)$ for six of our study sites.

We also computed the seasonality in rainfall, based on monthly rainfall data, and called this parameter SR. Specifically, we defined SR as

$\mathrm{SR}=\frac{\|\boldsymbol{m} r\|}{R}$

Where $\boldsymbol{m} r=\left(m r_{x}, m r_{y}\right)$, denotes the monthly rainfall vector defined like in Eq. (1) by

$m r_{x}=\sum_{i=0}^{11} R^{i} \cos (30 \times i), \quad m r_{y}=\sum_{i=0}^{11} R^{i} \sin (30 \times i)$

Here, $R^{i}$ is the monthly rainfall for month $i$ measured in $\mathrm{mm} / \mathrm{mo}$. Then, annual rainfall is $R=\sum_{i=0}^{11} R^{i}$, a variable that appears in Eq. (3).

To investigate the relative investment into reproduction versus photosynthesis, we computed the RL ratio, the investment into reproductive organs divided by leaf fall. 

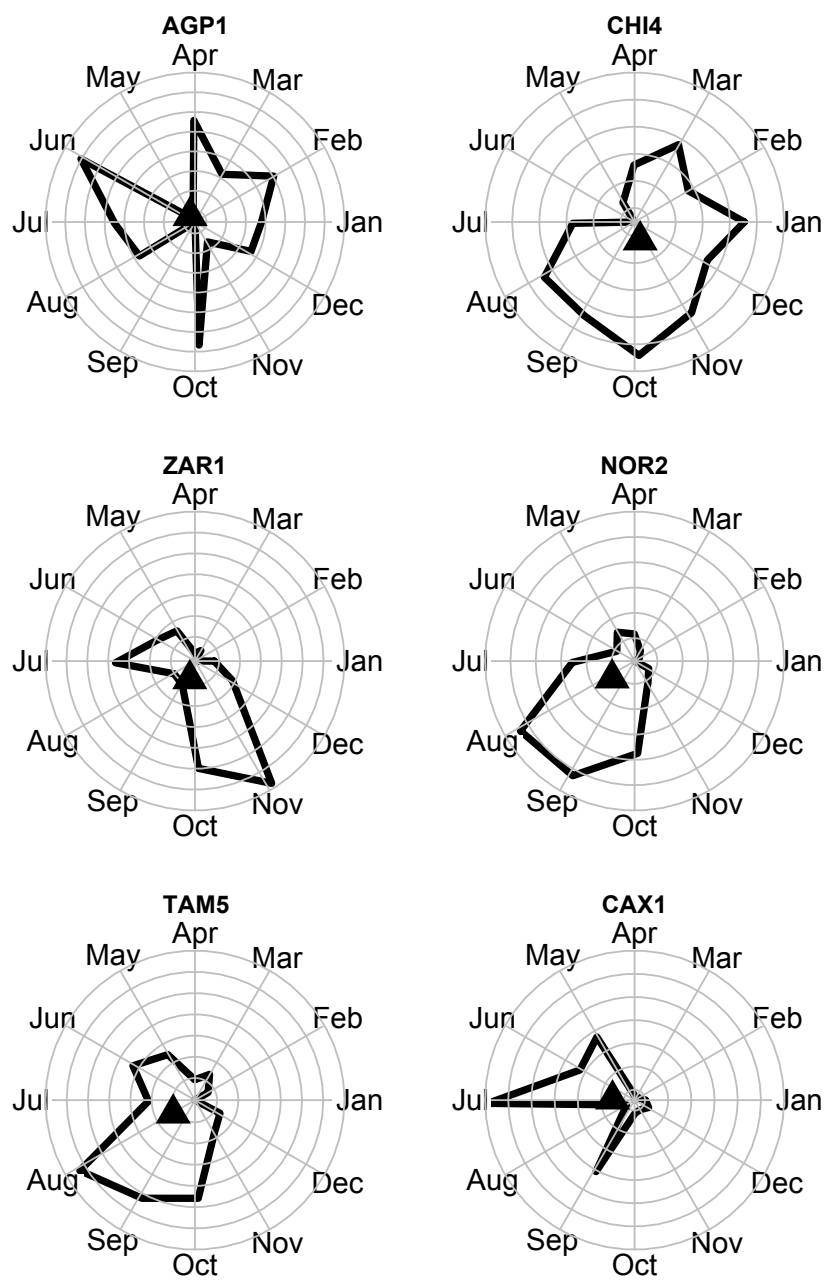

Fig. 1. Seasonality patterns for total litterfall at six sites (for site names, see Table 1). Thick lines delineate the envelope of monthly litterfall. The sites are ranked by increasing seasonality from left to right and top to bottom. Seasonality was measured using the equations reported in the Methods.

Hence a RL of 1 corresponds to an equal allocation into leaves and into reproductive organs. This excludes all nonphotosynthetic organs which make up non-reproductive litterfall (twigs and trash) and provides a firm baseline for comparison across sites.

\section{Results}

\subsection{Determinants of annual litterfall}

In old-growth tropical rainforests, which cover the vast majority of the area under study, litterfall averaged $8.61 \pm 1.91 \mathrm{Mg} \mathrm{ha}^{-1} \mathrm{yr}^{-1} \quad(n=52$, range: 5.19$12.47 \mathrm{Mg} / \mathrm{ha} / \mathrm{yr}$ ). We assessed Proctor's (1983) claim that one year of litterfall collection was enough to capture this variable. Of the 24 sites for which we had 2 years of data

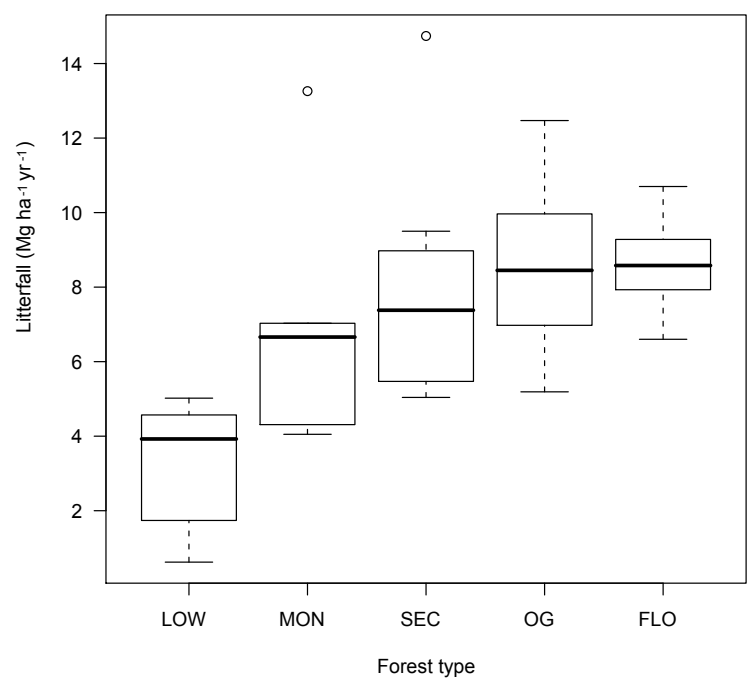

Fig. 2. Total annual litterfall in different forest types. LOW: shortstatured tropical forests (see Methods for a description), MON: montane tropical forests, SEC: secondary tropical forests, OG: oldgrowth tropical forests, FLO: partially flooded tropical forests. For each forest type, the thick horizontal lines represents the mean, the box represents the standard deviations (possibly asymmetrical), and the dotted line represents the $95 \%$ confidence intervals. Two outliers were detected, both above $12 \mathrm{Mg} \mathrm{ha}^{-1} \mathrm{yr}^{-1}$ (dots).

or more, mean interannual variability was found to be equal to $9.3 \%$ of the mean (range: $2 \%-20 \%$ ). Hence, one year of litterfall collection captures the long trend of litterfall within $10 \%$.

Annual litterfall was higher in flooded forests than in old-growth tropical forests (Fig. 2), with a mean of $8.89 \pm 1.42 \mathrm{Mg} \mathrm{ha}^{-1} \mathrm{yr}^{-1} \quad(n=10, \quad$ range: $6.6-$ 11.21 $\mathrm{Mg} \mathrm{ha}^{-1} \mathrm{yr}^{-1}$ ). Secondary forests had lower annual litterfall than old-growth tropical forests with a mean of $8.01 \pm 3.41 \mathrm{Mg} \mathrm{ha}^{-1} \mathrm{yr}^{-1} \quad(n=10$, range: 5.0114.74 $\left.\mathrm{Mg} \mathrm{ha}^{-1} \mathrm{yr}^{-1}\right)$. The outlying secondary forest (14.74 $\mathrm{Mg} \mathrm{ha}^{-1} \mathrm{yr}^{-1}$ ) was at the edge of the Mata de Piedade site, Atlantic rain forest of Brazil. Montane forests and low forests had lower mean annual litterfall (7.06 $\pm 3.72 \mathrm{Mg} \mathrm{ha}^{-1} \mathrm{yr}^{-1}$ and $3.01 \pm 1.67 \mathrm{Mg} \mathrm{ha}^{-1} \mathrm{yr}^{-1}$, respectively). Figure 3 shows the regional variation of litterfall across all the dataset (panel a) and restricted to old-growth forests (panel b).

Across forest types, annual litterfall showed no significant variation with total annual rainfall (Fig. 4). We excluded montane forests from this analysis because of the difficulty of estimating rainfall for these environments. With our analysis restricted to old-growth and flooded forests, the relationship between annual litterfall an annual rainfall was not significant ( $p=0.88$ and $p=0.23$, respectively). Secondary forests showed a negative relationship of annual litterfall with annual rainfall, but this trend was not significant $(p=0.18)$. 

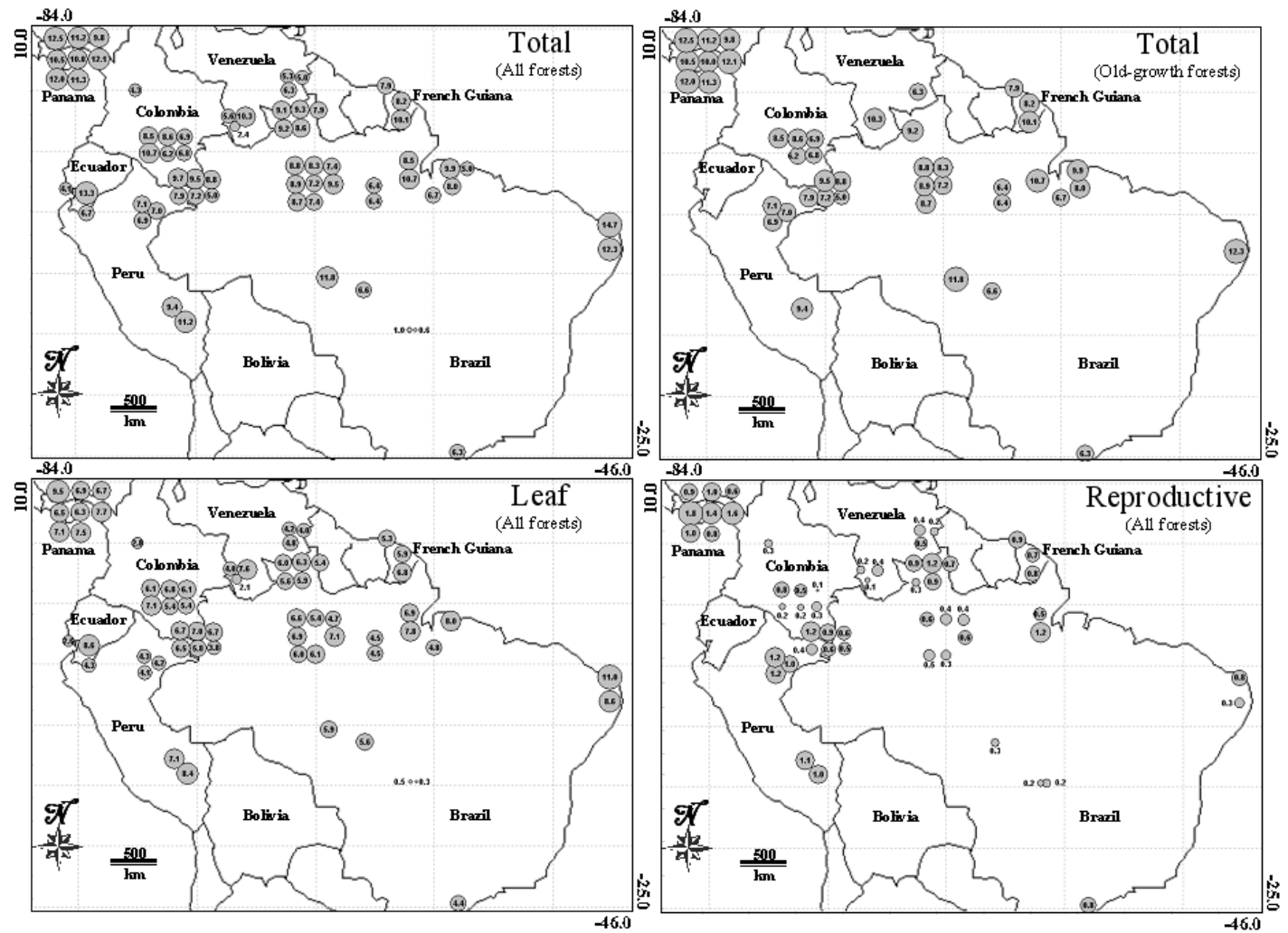

Fig. 3. Regional variation in litterfall. Variation in total litterfall across the sites (a), only in old-growth forests (b), variation in leaf fall (c) and variation in allocation into reproductive organs (d). All figures are in $\mathrm{Mg} \mathrm{ha}^{-1} \mathrm{yr}^{-1}$.

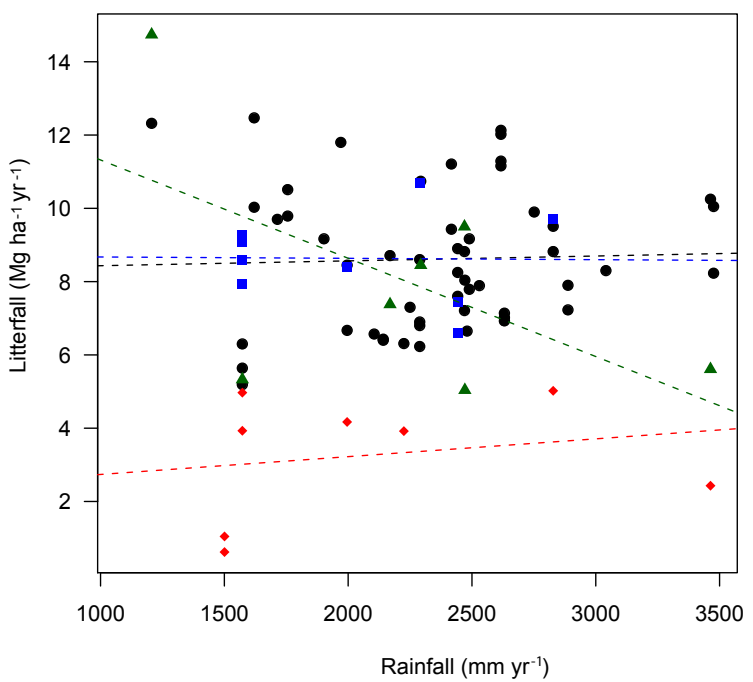

Fig. 4. Total annual litterfall versus annual rainfall for four lowland forest types. The four forest types are: old-growth tropical forests (black dots), flooded tropical forests (blue squares), secondary tropical forests (green triangles), and short-statured tropical forests (red diamonds). The dashed lines represent the least-square regression of total annual litterfall versus annual rainfall at the four forest sites. None of these regressions were significant.

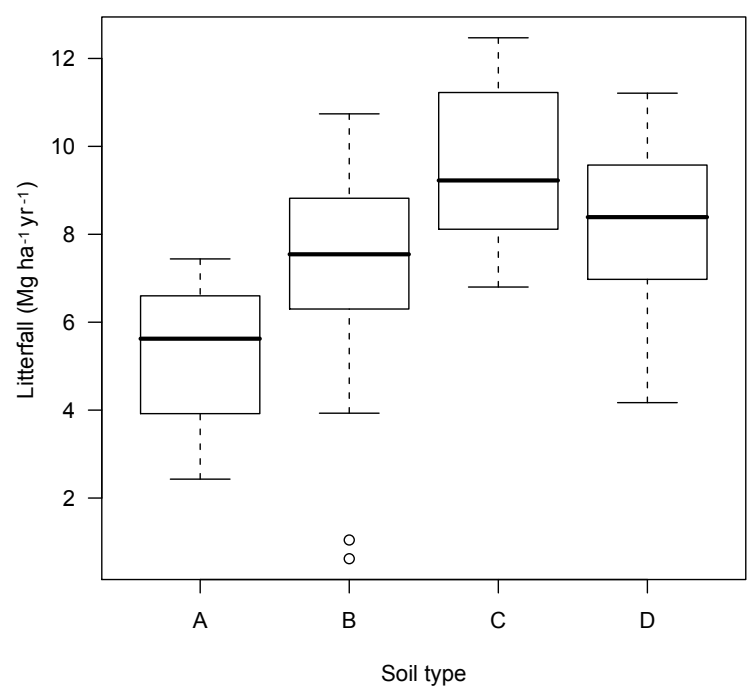

Fig. 5. Total annual litterfall on different soil types. Soil types are based on the WRB taxonomy (for more details, see Methods and Quesada, 2008). Soil types are as follows. A: arenosols/podzols; B: ferrasols; C: acrisols/plinthosols/alisols); D: cambisols/leptosols/histosols/gleysols/fluvisols. The notations of this figure are the same as in Fig. 2. 


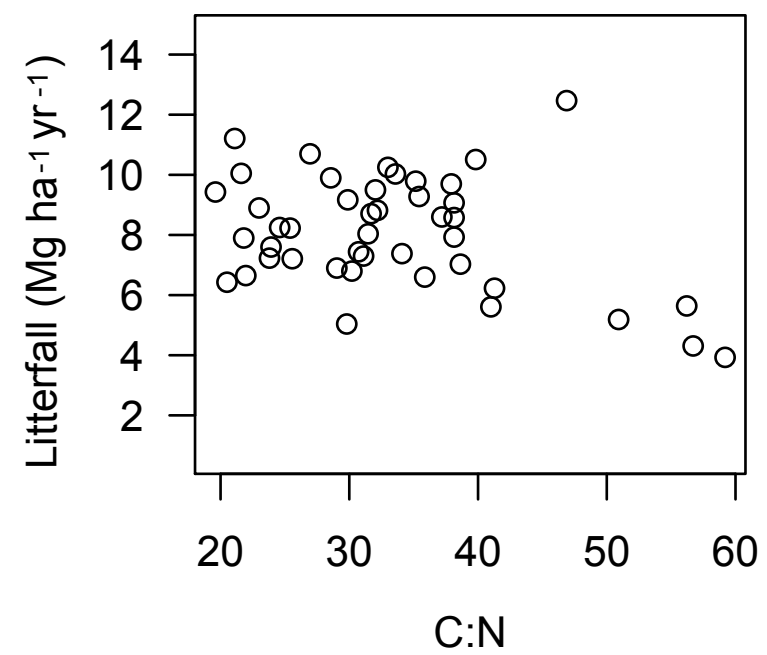

Fig. 6. Total annual litterfall versus $\mathrm{C}: \mathrm{N}$ ratio. The correlation is not significant (Kendall rank test: $\mathrm{p}$-value $=0.16$ ).

We limited our analysis of annual litterfall versus soil type to old-growth moist lowland rainforests (Fig. 5). The poor soils are found in group A (including white sand soils), and litterfall was significantly lower than in other soil types (5.27 $\pm 1.86 \mathrm{Mg} \mathrm{ha}^{-1} \mathrm{yr}^{-1}, n=6$ ). Ferralsols (group B) also supported a forest producing less litterfall annually (7.13 $\left.\pm 2.53 \mathrm{Mg} \mathrm{ha}^{-1} \mathrm{yr}^{-1}, n=26\right)$.

A similar analysis was performed by using the Redfield ratio $\mathrm{C}: \mathrm{N}$ rather than soil types as independent variables (Ågren, 2008). Nitrogen-deprived plants have a large C:N ratio. Litterfall was found to decline albeit not significantly with $\mathrm{C}: \mathrm{N}$ across the entire dataset (Fig. 6, Kendall rank test $p=0.16, n=44)$.

\subsection{Determinants of litterfall seasonality}

Across all plots, the litterfall seasonality index SL, computed from 47 datasets, was of 0.166 , indicating a mild seasonality of litterfall.

Litterfall seasonality was highest in small-statured forest sites (LOW), and lowest in montane and flooded forest sites (respectively MON and FLO, see Fig. 7). Litterfall seasonality did not depend on annual rainfall either across all datasets, or across old-growth forest sites only (in both cases, $p>0.4$, results not shown). Litterfall seasonality did not depend on soil type either.

Next we explored whether litterfall seasonality SL was related with the rainfall seasonality index SR (see the Methods section). We found a significantly positive relationship between litterfall seasonality and rainfall seasonality across all plots ( $p=0.02, n=47$, Fig. 8). This result also held when the analysis was restricted to old-growth forests $(p=0.05$, $n=27)$.

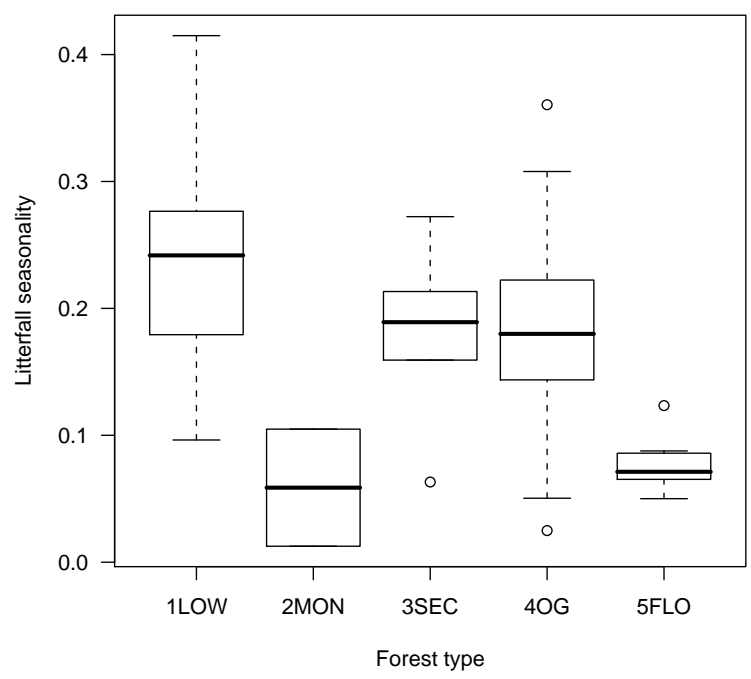

Fig. 7. Litterfall seasonality index SL (see Methods) in different forest types. The notations are the same as in Fig. 2.

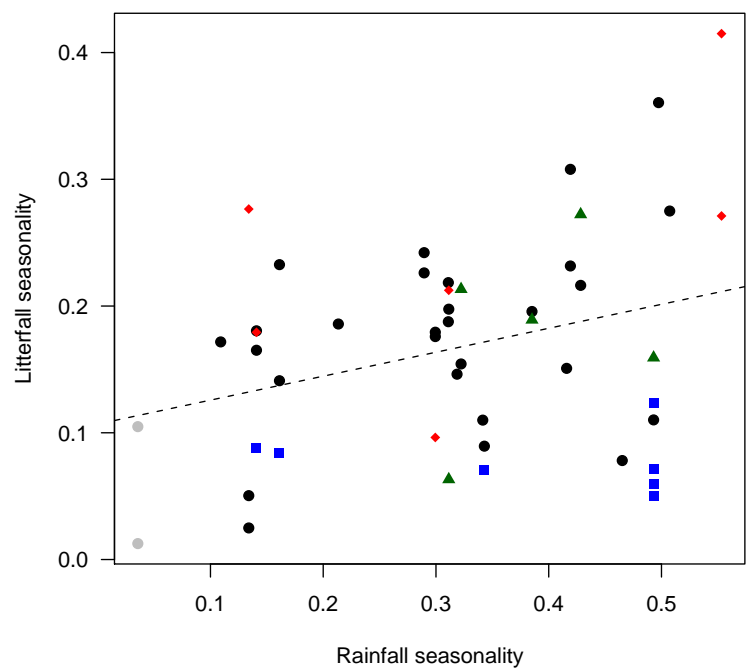

Fig. 8. Litterfall seasonality index SL versus rainfall seasonality index SR. The dashed line represents a regression across all points $\left(r^{2}=0.10, p=0.02\right)$. Color codes show forest types as in Fig. 4 .

\subsection{Carbon allocation in fast turnover plant organs}

Finally, we asked how much carbon is stored in leaves and in reproductive organs. Across the dataset, $70.8 \pm 8.5 \%$ of the litterfall was allocated to leaves $(n=74$, range $43.1 \%$ $88.4 \%$ ). Mean leaf fall was $5.74 \pm 1.83 \mathrm{Mg} \mathrm{ha}^{-1} \mathrm{yr}^{-1}$. Likewise, $8.9 \pm 5.6 \%$ of the litterfall was allocated to reproductive organs $(0.8 \%-18 \%)$. Mean allocation into reproductive organs was $0.69 \pm 0.40 \mathrm{Mg} / \mathrm{ha} / \mathrm{yr}$. Notice however that some of these reproductive organs are often eaten before they fall, hence our figure may be an underestimate. 
Next we computed the RL ratio for our sites (investment into reproductive organs divided by leaf litterfall). Across sites, this ratio ranged between 0.008 and 0.89 and was $0.135 \pm 0.119$ on average (note that a ratio of 1 corresponds to an equal allocation into leaves and into reproductive organs). We did not find significant differences in the RL among forest types, except secondary forests where RL was significantly smaller $(0.07 \pm 0.018)$.

The RL ratio varied across soil types. It was smallest on group-A soils ( $\mathrm{RL}=0.081 \pm 0.036, n=5$ ), in acidic group-C soils $(\mathrm{RL}=0.11 \pm 0.06, n=22)$, in group- $\mathrm{B}$ ferralsols ( $\mathrm{RL}=0.17 \pm 0.21 n=16)$, and finally in richer group-D soils $(0.18 \pm 0.07, n=11)$. Given that frugivore activity also correlates positively with nutrients, the actual RL ratios probably increase more steeply than this with soil nutrients. This suggests that plants growing on rich soils invest proportionally more into reproduction than into photosynthesis.

\section{Discussion}

Assuming that litterfall biomass contains $47 \%$ of carbon (cross-site mean taken from Fyllas et al., 2009), the total annual litterfall corresponds to a mean of $8.0 \mathrm{Mg} \mathrm{ha}^{-1} \mathrm{yr}^{-1}$ in old-growth tropical forests. This is in line with previous estimates of Amazon-wide allocation of carbon into the fast turnover carbon pool (Clark et al., 2001). If the overall figure of NPP around $25.6 \mathrm{Mg} \mathrm{ha}^{-1} \mathrm{yr}^{-1}$ is valid for Amazonian forests (Aragão et al., 2009), then, about a third of total NPP is invested into leaves, twigs and reproductive organs. The largest fraction of soft tissue allocation is invested into photosynthesis (ca. 71\%). Another 9\% is invested into reproduction. Following Clark et al. (2001), we reemphasize that the estimates of litterfall reported here do not include large branches. Other methods may be used to assess how much carbon is released by branch falls, and this flux ranges between 0.8 and $3.6 \mathrm{Mg} \mathrm{ha}^{-1} \mathrm{yr}^{-1}$ (Chambers et al., 2001; Nepstad et al., 2002).

Most of the NPP eventually contributing to fine litterfall is allocated to leaves. Because leaf fall was estimated around $5.6 \mathrm{Mg} \mathrm{ha}^{-1} \mathrm{yr}^{-1}$ in the field, the stocks of photosynthetically active material available in the ecosystem may be estimated through two independent methods. First, the stock of leaves at any one time $f B$ is related to fNPP through the mean lifetime of leaves, denoted by $\tau: \tau=f B / \mathrm{fNPP}$. This parameter $\tau$ can be estimated directly for selected species, and it varies between 6 months for secondary moist tropical forests ( $n=20$, Coley, 1988), and 25 months for old-growth tropical forests on poor soils $(n=23$, Reich et al., 2004). Taking an average value of $\tau=1 \mathrm{yr}$, the stock of leaf biomass is estimated at $2.8 \mathrm{MgC} \mathrm{ha}^{-1}$, or $280 \mathrm{gC} \mathrm{m}^{-2}$. Alternatively, assuming that the leaf area index of Amazonian forests is close to $5.4 \mathrm{~m}^{2} / \mathrm{m}^{2}$ (Malhi et al., 2009; Patiño et al., unpublished data; it may reach up to $7 \mathrm{~m}^{2} / \mathrm{m}^{2}$, see Clark et al., 2008), and that mean leaf-mass area (LMA) is around $47 \mathrm{gC} \mathrm{m}^{-2}$ (crosssite mean taken from Fyllas et al., 2009), then leaf biomass should be $254 \mathrm{gC} \mathrm{m}^{-2}$. These two estimates tightly bracket the leaf biomass stocks in tropical rain forests. They also provide a consistency check for some of the lesser known variables in Amazonian rainforests (mean leaf lifetime and leaf area index).

Secondary forests showed a peculiar signal compared with old-growth forests. Although the total annual litterfall was comparable between secondary forests and old-growth forests, the former were less seasonal, and they invested less in reproduction than in photosynthesis. Since secondary tropical forests are likely to cover an ever larger area than today, and will remain in secondary status for a long time (Chazdon, 2003; Feldpausch et al., 2005, 2007), it is critical to account for this in global carbon cycle models.

There was a positive correlation between total litterfall and soil richness. This pattern may be underestimated in our analysis because herbivory is more active in the most fertile forests (Gentry and Emmons, 1987). Litterfall is already highest in forests growing on fertile soils (Fig. 5), and the amount of missed litterfall is difficult to quantify. Also, in many Amazonian forests, palms are an important fraction of the flora, and these palms also contribute to number of bias to litterfall as estimated by litter traps. Large palms tend to trap litter in their crown hence reducing the amount of litter falling to the ground (Alvarez-Sánchez and Guevara, 1999). Furthermore, many palm species have big leaves that tend to be discarded in litter trap measurements, since they are considered as coarse debris. These effects add up in western Amazonian forests, and it would therefore be important to develop different methods for litter collection in these forests. Then the positive relationship between litterfall and soil richness (see Fig. 5) may be linear rather than curvilinear.

We found a weak but significant correlation between litterfall seasonality and rainfall seasonality. This may be explained by limitations in our dataset, or by biological mechanisms. In the former class, several unpublished datasets span unusual climatic years, such as the intense 2005 drought, and they may therefore be not representative of the long-term trend in seasonality. In the latter category of explanations, it is known that leaves are not shed or flushed only in response to variation in rainfall. Recently developed methods may be used to estimate, even though indirectly, the large scale variation in leaf coverage seasonality. Myneni et al. (2007) used remote sensing imagery techniques to show how the seasonality in green leaf cover (leaf area index, or LAI) varies across the Amazon. They also sought for causal explanations for this variation. Specifically, they suggested that LAI was driven by the seasonality in solar radiation, rather than in rainfall. Indeed, solar radiation may be a foremost trigger for the flushing of new leaves during the dry season (see Wright and van Schaik, 1994), but also of leaf abscission, leading to concerted leaf fall. Phenological models (Morin and Chuine, 2005) remain poorly developed for tropical trees (Sakai, 2001), and this important challenge is ahead of us. 
Finally, our results shed light on carbon allocation strategies of tropical trees. We have shown that in poor soils, and especially in phosphorus-deprived environments, forests as a whole tend to invest less into the construction of reproductive organs relative to photosynthesis. This suggests that allocation into leaves (hence photosynthesis) is the priority for plants, but when resources are well supplied the excess in resources is made available for reproduction. Also, the plants of poor-soil communities seem to converge toward a low growth rate, low mortality rate and infrequent reproduction, a classic example of habitat filtering (Weiher and Keddy, 1999). The pattern we uncovered should however be considered critically. Tropical forest reproduction is often characterized by infrequent events of mast-flowering, hence the RL ratio should show a high interannual variability. For instance, at the Nouragues site, one of the dominant tree families, the Chrysobalanaceae has a mast-fruiting strategy, and these species have only fruited once between 2001 and 2008 (Norden et al., 2007). Hence, it would be essential to rely on long-term monitoring programs to accurately measure RL. Finally, fruit production is clearly underestimated in palmrich forests of western Amazon. More refined tests of this hypothesis should be based on more thorough and appropriate measurements of resources available to plants.

Acknowledgements. Some of the unpublished datasets were funded through the European Union funded PAN-AMAZONIA programme, a UK Natural Environment Research Council (NERC) grant (NER/A/S/2003/00608/2) to Y. Malhi, and continuous funding by the French CNRS (in part through the Amazonie program). We thank Angela Rozas Dávila, Judith Huamán Ovalle, Marlene Mamani Solórzano, Silverio Tera-Akami, Alfredo Andoke, José Agustín López, Germán Mejía, Eugenio Sánchez, Arcesio Pijachi and Hernán Machoa for their help in the field, and Carlos A. Quesada and Jon Lloyd for their help with the soil classification at our sites, and for useful comments on this manuscript.

Edited by: J. Lloyd

\section{References}

Ågren, G. I.: Stoichiometry and nutrition of plant growth in natural communities, Ann. Rev. Ecol. Evol. Syst., 39, 153-170, 2008.

Alvarez-Sánchez, J. and Guevara, S.: Litter interception on Astrocaryum mexicanum Lieb. (Palmae) in a tropical rain forest, Biotropica, 31, 89-92, 1999

Aragão, L. E. O. C., Malhi, Y., Metcalfe, D. B., Silva-Espejo, J. E., Jiménez, E., Navarrete, D., Almeida, S., Costa, A. C. L., Salinas, N., Phillips, O. L., Anderson, L. O., Alvarez, E., Baker, T. R., Goncalvez, P. H., Huamán-Ovalle, J., MamaniSolórzano, M., Meir, P., Monteagudo, A., Patiño, S., Pe/uela, M. C., Prieto, A., Quesada, C. A., Rozas-Dávila, A., Rudas, A., Silva Jr., J. A., and Vásquez, R.: Above- and belowground net primary productivity across ten Amazonian forests on contrasting soils, Biogeosciences, 6, 2759-2778, 2009, http://www.biogeosciences.net/6/2759/2009/.
Barbosa, R. A. and Fearnside, P. M.: Carbon and nutrient flows in an Amazonian forest: Fine litter production and composition at Apiau, Roraima, Brazil. Trop. Ecol., 37, 115-125, 1998.

Barlow, J., Gardner, T. A., Ferreira, L. V., and Peres, C. A.: Litter fall and decomposition in primary, secondary and plantation forests in the Brazilian Amazon, Forest Ecol. Manage., 247, 9197, 2007.

Brando, P. M., Nepstad, D. C., Davidson, E. A., Trumbore, S. E., Ray, D., and Camargo, P.: Drought effects on litterfall, wood production and belowground carbon cycling in an Amazonian forest: result of a throughfall reduction experiment, Philos. T. Roy. Soc. B, 363, 1839-1848, 2008.

Bray, J. R. and Gorham, E.: Litter production in forests of the world, Adv. Ecol. Res., 2, 101-157, 1964.

Chambers, J. Q., dos Santos, J., Ribeiro, R. J., and Higuchi, N.: Tree damage, allometric relationships, and above-ground net primary production in central Amazon forest, Forest Ecol. Manage., 152, 73-84, 2001.

Chazdon, R. L.: Tropical forest recovery: legacies of human impact and natural disturbances, Persp. Plant Ecol. Evol. Syst., 6, 51-71, 2003.

Chuyong, G. B., Newbery, D. M., and Songwe, N. C.: Litter nutrients and retranslocation in a central African rain forest dominated by ectomycorrhizal trees, New Phytol., 148, 493-510, 2000.

Clark, D. A., Brown, S., Kicklighter, D. W., Chambers, J. Q., Thomlinson, J. R., Ni, J., and Holland, E. A.: Net primary production in tropical forests: an evaluation and synthesis of existing field data, Ecol. Appl., 11, 371-384, 2001.

Clark, D. B., Olivas, P. C., Oberbauer, S. F., Clark, D. A., and Ryan, M. G.: First direct landscape-scale measurement of tropical rain forest Leaf Area Index, a key driver of global primary productivity, Ecol. Lett., 11, 163-172, 2008.

Coley, P. D.: Effects of plant growth rate and leaf lifetime of the amount and type of anti-herbivore defense, Oecologia, 74, 531536, 1988.

Cuevas, E. and Medina, E.: Nutrient dynamics within Amazonian forest ecosystems. I. Nutrient flux in fine litterfall and efficiency in nutrient utilization, Oecologia, 68, 4466-4472, 1986.

Del Grosso, S., Parton, W., Stohlgren, T., Zheng, D. L., Bachelet, D., Prince, S., Hibbard, K., and Olson, R.: Global potential net primary production predicted from vegetation class, precipitation, and temperature, Ecology, 89, 2117-2126, 2008.

Dezzeo, N. and Chacon, N.: Litterfall and nutrient input in undisturbed and adjacent fire disturbed forests of the Gran Sabana, Southern Venezuela, Interciencia, 31, 894-899, 2006.

Dantas, M. and Phillipson, J.: Litterfall and litter nutrient content in primary and secondary Amazonian "terra firme" rain forest, J. Trop. Ecol., 5, 27-36, 1989.

Esser, G., Lieth, H. F. H., Scurlock, J. M. O., and Olson, R. J.: Worldwide estimates and bibliography of net primary productivity derived from pre-1982 publications. ORNL/TM-13485. Oak Ridge National Laboratory, Oak Ridge, Tennessee, USA, 1997.

Feldpausch, T. R., Riha, S., Fernandes, E. C. M., and Wandelli, E. V.: Development of forest structure and leaf area in secondary forests regenerating on abandoned pastures in Central Amazonia, Earth Interactions, 9, 1-22, 2005.

Feldpausch, T. R., Prates-Clark, C. C., Fernandes, E. C. M., and Riha, S. J.: Secondary forest growth deviation from chronosequence predictions in central Amazonia, Glob. Change Biol., 13, 
967-979, 2007.

Fittkau, E. J. and Klinge, H.: On biomass and trophic structure of the Central Amazonian rain forest ecosystem, Biotropica, 5, 214, 1973.

Fyllas, N. M., Patiño, S., Baker, T. R., Bielefeld Nardoto, G., Martinelli, L. A., Quesada, C. A., Paiva, R., Schwarz, M., Horna, V., Mercado, L. M., Santos, A., Arroyo, L., Jiménez, E. M., Luizão, F. J., Neill, D. A., Silva, N., Prieto, A., Rudas, A., Silviera, M., Vieira, I. C. G., Lopez-Gonzalez, G., Malhi, Y., Phillips, O. L., and Lloyd, J.: Basin-wide variations in foliar properties of Amazonian forest: phylogeny, soils and climate, Biogeosciences, 6 , 2677-2708, 2009, http://www.biogeosciences.net/6/2677/2009/.

Gentry, A. H. and Emmons, L. H.: Geographical variation in fertility, phenology, and composition of the understory of Neotropical forests, Biotropica, 19, 216-227, 1987

Grace, J.: Understanding and managing the global carbon cycle, J. Ecol., 92, 189-202, 2004.

Hättenschwiler, S., Aeschlimann, B., Coûteaux, M.-M., Roy, J., and Bonal, D.: High variation in foliage and leaf litter chemistry among 45 tree species of a neotropical rainforest community, New Phytol., 179, 165-175, 2008.

Keller, M., Alencar, A., Asner, G. P., Braswell, B., Bustamente, M., Davidson, E., Feldpausch, T., Fernandes, E., Goulden, M., Kabat, P., Kruijt, B., Luizão, F., Miller, S., Markewitz, D., Nobre, A. D., Nobre, C. A., Priante Filho, N., da Rocha, H., Silva Dias, P., von Randow, C., and Vourlitis, G. L.: Ecological research in the Large-Scale Biosphere-Atmosphere experiment in Amazonia: early results, Ecol. Appl., 14, S3-S16, 2004.

Klinge, H. and Rodrigues, W. A.: Litter production in an area of Amazonian terra firme forest, Amazoniana, 1, 287-310, 1968.

Leigh Jr., E. G.: Tropical Forest Ecology. A View from Barro Colorado Island, Oxford University Press, Oxford, 245 pp., 1999.

Lips, J. M. and Duivenvoorden, J. F.: Fine litter input to terrestrial humus forms in Colombian Amazonia, Oecologia, 108, 138150, 1996.

Luizão, R. C. C., Luizão, F. J., Paiva, R. Q., Monteiro, T. F., Sousa, L. S., and Kruijt, B.: variation of carbon and nitrogen cycling processes along a topographic gradient in a central Amazonian forest, Glob. Change Biol., 10, 592-600, 2004.

Luizão, F. J.: Litter production and mineral element input to the forest floor in a Central Amazonian forest, GeoJournal, 19, 407$417,1989$.

Luyssaert, S., Inglima, I., Jung, M., Richardson, A. D., M. Reichstein M., et al.: $\mathrm{CO}_{2}$ balance of boreal, temperate, and tropical forests derived from a global database, Glob. Change Biol., 13, 2509-2537, 2007.

Malhi, Y., Phillips, O. L., Baker, T. R., et al.: An international network to understand the biomass and dynamics of Amazonian forests (RAINFOR), J. Veg. Sci., 13, 439-450, 2002.

Malhi, Y., Baker, T. R., Phillips, O. L., et al. The above-ground coarse wood productivity of 104 Neotropical forest plots, Glob. Change. Biol., 10, 563-591, 2004.

Malhi, Y., Aragão, L. E. O. C., Metcalfe, D. B., Paiva, R., Quesada, C. A., Almeida, S., Anderson, L., Brando, P., Chambers, J. Q., da Costa, A. C. L., Hutyra, L. R., Oliveira, P., Patiño, S., Pyle, L. H., Robertson, A. L., and Teixeira, L. M.: Comprehensive assessment of carbon productivity, allocation and storage in three Amazonian forests, Glob. Change. Biol., 15, 1255-1274, 2009.

Martius, C., Höfer, H., Garcia, M. V. B., Römbke, J., and Hana- garth, W.: Litter fall, litter stocks and decomposition rates in rainforest and agroforestry sites in central Amazonia, Nutr. Cycl. Agroecosyst., 68, 137-154, 2004.

McGroddy M. E., Daufresne, T., and Hedin, L. O.: Scaling of C:N:P stoichiometry in forests worldwide: Implications of terrestrial Redfield-type ratios, Ecology, 85, 2390-2401, 2004.

Morães, R. M., Carvalho, W. B., and Struffaldy de Vuono, Y.: Litterfall and litter nutrient content in two Brazilian tropical forests, Rev. Bras. Bot., 22, 9-16, 1999.

Morin, X. and Chuine, I.: Sensitivity analysis of the tree distribution model PHENOFIT to climatic input characteristics: implications for climate impact assessment, Glob. Change Biol., 9, 1493-1503, 2005.

Myneni, R. B., Yanga, W., Nemani, R. R., Huete, A. R., Dickinsone, R. E., Knyazikhina, Y., Didan, K., Fu, R., Negron Juarez, R. I., Saatchi, S. S., Hashimoto, H., Ichii K., Shabanov, N. V., Tana, B., Ratana, P., Privette, J. L., Morisette, J. T., Vermote, E. F., Roy, D. P., Wolfe, R. E., Friedl, M. A., Running, S. W., Votava, P., El-Saleous, N., Devadiga, S., Su, Y., and Salomonson, V. V.: Large seasonal swings in leaf area of Amazon rainforests, P. Natl. Acad. Sci., 104, 4820-4823, 2007.

Nebel, G., Dragsted, J., and Vega, A. S.: Litter fall, biomass and net primary production in flood plain forests in the Peruvian Amazon, Forest Ecol. Manage., 150, 93-102, 2001.

Nepstad, D. C., Moutinho, P., Dias-Filho, M. B., Davidson, E., Cardinot, G., Markewitz, D., Figueiredo, R., Vianna, N., Chambers, J., Ray, D., Guerreiros, J. B., Lefebvre, P., Sternberg, L., Moreira, M., Barros, L., Ishida, F. Y., Tohlver, I., Belk, E., Kalif, K., and Schwalbe, K.: The effects of partial throughfall exclusion on canopy processes, aboveground production, and biogeochemistry of an Amazon forest, J. Geophys. Res. Atmos., 107, 8085, doi:10.1029/2001JD000360, 2002.

New, M., Hulme, M., and Jones, P.: Representing twentieth century space-time climate variability. Part I. Development of a 19611990 mean monthly terrestrial climatology, J. Climate, 12, 829856, 1999.

Norden, N., Chave, J., Belbenoît, P., Caubère, A., Châtelet, P., Forget, P.-M., and Thébaud, C.: Mast fruiting is a frequent strategy in woody species of Eastern South America, PLoS ONE, 2, e1079, doi:10.1371/journal.pone.0001079, 2007.

Priess, J. A., Then, Ch., and Fölster, H.: Litter and fine-root production in three types of tropical premontane rain forest in SE Venezuela, Plant Ecol., 143, 171-187, 1999.

Proctor, J.: Tropical forest litterfall. I. Problems of litter comparison, in: Tropical rain forest: ecology and management, edited by: Sutton, S. L., Whitmore, T. C., and Chadwick, A. C., Blackwell, Oxford, UK, 267-273, 1983.

Puig, H., Riéra, B., and Lescure, J.-P.: Phytomasse et productivité, Bois Forêts Trop., 220, 25-32, 1983.

Quesada, C. A.: Soil Vegetation Interactions Across Amazonia, Unpublished PhD dissertation, University of Leeds, 2009.

Quesada, C. A., Lloyd, J., Schwarz, M., Patiño, S., Baker, T. R., Czimczik, C., Fyllas, N. M., Martinelli, L., Nardoto, G. B., Schmerler, J., Santos, A. J. B., Hodnett, M. G., Herrera, R., Luizão, F. J., Arneth, A., Lloyd, G., Dezzeo, N., Hilke, I., Kuhlmann, I., Raessler, M., Brand, W. A., Geilmann, H., Moraes Filho, J. O., Carvalho, F. P., Araujo Filho, R. N., Chaves, J. E., Cruz Junior, O. F., Pimentel, T. P., and Paiva, R.: Chemical and physical properties of Amazon forest soils in relation 
to their genesis, Biogeosciences Discuss., 6, 3923-3992, 2009, http://www.biogeosciences-discuss.net/6/3923/2009/.

Reich, P. B., Uhl, C., Walters, M. B., Prugh, L., and Ellsworth, D. S.: Leaf demography and phenology in Amazonian rain forest: a census of 40000 leaves of 23 tree species, Ecol. Monogr., 74, 3-23, 2004.

Röderstein, M., Hertel, D., and Leuschner, C.: Above- and belowground litter production in three tropical montane forests in Southern Ecuador, J. Trop. Ecol., 21, 483-492, 2005.

Sakai, S.: Phenological diversity in tropical forests, Pop. Ecol., 43, 77-86, 2001.

Santiago, L. S., Schuur, E. A. G., and Silvera, K.: Nutrient cycling and plant-soil feedbacks along a precipitation gradient in Lowland Panama, J. Trop. Ecol., 21, 461-470, 2005.

Schessl, M., Luiz da Silva, W., and Gottsberger, G.: Effects of fragmentation on forest structure and litter dynamics in Atlantic rainforest in Pernambuco, Brazil. Flora, 203, 215-228, 2008.

Schuur, E. A. G.: Productivity and global climate revisited: the sensitivity of tropical forest growth to precipitation, Ecology, 84, 1165-1170, 2003.

Scott, D. A., Proctor, J., and Thompson, J.: Studies on a lowland evergreen rain forest on Maraca island, Roraima, Brazil. II. Litter and nutrient cycling, J. Ecol., 80, 705-717, 1992.

Selva, E. C., Couto, E. G., Johnson, M. S., and Lehmann, J.: Litterfall production and fluvial export in headwater catchments of the southern Amazon, J. Trop. Ecol., 23, 329-335, 2007.

Silva, C. J., Sanches, L., Bleich, M. E., Lobo, F. A., and Nogueira, J. S.: Produção de serrapilheira no cerrado e floresta de transição Amazônia-Cerrado do Centro-Oeste Brasileiro, Acta Amazonica, 37, 543-548, 2007.
Sizer, N. C., Tanner, E. V. J., and Kossmann Ferraz, I. D.: Edge effects on litterfall mass and nutrient concentrations in forest fragments in central Amazonia, J. Trop. Ecol., 16, 853-863, 2000.

Smith, K., Gholz, H. L., and Oliveira, F. A.: Litterfall and nitrogenuse efficiency of plantations and primary forest in the eastern Brazilian Amazon, Forest Ecol. Manage., 109, 209-220, 1998.

Sombroek W. G.: Amazon land forms and soils in relation to biological diversity, Acta Amazonica, 30, 81-100, 2000.

Vasconcelos, H. L. and Luizão, F. L.: Litter production and litter nutrient concentrations in a fragmented Amazonian landscape, Ecol. Appl., 14, 884-892, 2004.

Veneklaas, E. J.: Litterfall and nutrient fluxes in two montane tropical rain forests, Colombia, J. Trop. Ecol., 7, 319-336, 1991.

Weiher, E. and Keddy, P. A.: Ecological assembly rules: perspectives, advances, retreats, Cambridge University Press, Cambridge, UK, 1999.

WRB World Reference Base for Soil Resources: A framework for international classification, correlation and communication, World Soil Resources Report 103, FAO, Rome, 2006.

Wright, S. J. and van Schaik, C. P.: Light and the phenology of tropical trees, Am. Nat., 143, 192-199, 1994.

Zimmerman, J. K., Wright, S. J., Calderon, O., Aponte Pagan, M., and Paton, S.: Flowering and fruiting phenologies of seasonal and aseasonal neotropical forests: the role of annual changes in irradiance, J. Trop. Ecol., 23, 231-251, 2007. 